\title{
Balanced Joint Progressively Hybrid Type-I Censoring Samples in Estimating the Lifetime Lomax Distributions
}

\author{
Nada M. Alfaer (iD) and Hassan M. Aljohani $(D$ \\ Department of Mathematics \& Statistics, College of Science, Taif University, Taif, Saudi Arabia \\ Correspondence should be addressed to Nada M. Alfaer; nmfaer@tu.edu.sa
}

Received 28 March 2021; Accepted 26 April 2021; Published 1 June 2021

Academic Editor: Ahmed Mostafa Khalil

Copyright (c) 2021 Nada M. Alfaer and Hassan M. Aljohani. This is an open access article distributed under the Creative Commons Attribution License, which permits unrestricted use, distribution, and reproduction in any medium, provided the original work is properly cited.

\begin{abstract}
The comparative life testing for products from different production lines under joint censoring schemes has received some attention over the past few years. Mondal and Kundu recently used the balanced joint progressive type-II censoring scheme to discuss the comparative exponential and Weibull populations. This paper implements the balanced censoring scheme with a hybrid progressive type-I censoring scheme known as a balanced joint progressive hybrid type-I censoring scheme (BJPHCS). The life Lomax products' model formulation from two different lines of production with BJPHCS is discussed. The model parameters are estimated under maximum likelihood estimation for point and the corresponding asymptotic confidence intervals. Under independent gamma priors, the Bayes estimators and associated credible intervals are obtained with the help of MCMC technique. The validity of the theoretical results developed in this paper for estimation problems is discussed through numerical example and Monte Carlo simulation study, which report the estimators' quality. Finally, we give a brief comment describing the numerical results.
\end{abstract}

\section{Introduction}

The quality of any life product has required putting some product units under a life testing experiment, and the life data may be complete or censoring. The test cost and time determine a suitable method that is used to obtain the data. Type-I and type-II censoring schemes are presented as the oldest censoring schemes. The experiment is run to prefixed time in type-I censoring scheme, and the number of failed units is random. However, the experiment is run to prefixed number of failures in the Type-II censoring scheme with a random experiment time. In practice, if we need to remove units at any time of the experiment for engineering, clinical studies, or other purposes, then a progressive censoring scheme is applied to improve productivity while keeping a high level; see, for more details, [1-4]. The joint cases of typeI and type-II or progressive type-I and progressive type-II are called hybrid censoring schemes.

Suppose $n$ independent units are randomly selected from the product to put under test and the priors, integer $m$, ideal test time $\tau$, and censoring scheme $\mathbf{R}=\left\{R_{1}, R_{2}, \ldots, R_{m}\right\}$ such as $n=$ $m+\sum_{i=1}^{m} R_{i}$ are determined. At each failure time $T_{i}, R_{i}$ survival units are removed from the test, where $i=1,2, \ldots, m$. In progressive hybrid type-I censoring scheme, the experiment is terminated at $\min \left(\tau, T_{m}\right)$, but, in a progressive hybrid type-II censoring scheme, the experiment is terminated at $\max \left(\tau, T_{m}\right)$.

In particular, when the product comes from different production lines, a joint censoring scheme appears as a suitable censoring scheme, and the obtaining data are used to determine the relative merits of life products in competing duration. Therefore, suppose two lines of production $A_{1}$ and $A_{2}$ produced the same product under the same conditions. Suppose two independent samples $\kappa_{1}$ and $\kappa_{1}$ are selected from the lines $A_{1}$ and $A_{2}$, respectively, to be put simultaneously under life testing experiment. In the literature, joint censoring has been discussed in $[5,6]$. The classical estimation is discussed in $[7,8]$. Bayes estimation is presented by [9] and recently by [10-12].

For jointly progressive hybrid type-I censoring scheme, the total sample with size $\left(\kappa_{1}+\kappa_{2}\right)$ which is collected from the 
lines $A_{1}$ and $A_{2}$, where $\kappa_{1}$ from $A_{1}$ and $\kappa_{2}$ from $A_{2}$, are put under the test. Then, the number of failure units $m$, the censoring scheme $\underline{\mathbf{R}}=\left\{R_{1}, R_{2}, \ldots, R_{m}\right\}$, and the ideal test time $\tau$ is given at the prior of the experiment. The failure time and the corresponding unit type (from $A_{1}$ or $A_{2}$ ), say $\left(T_{i}, w_{i}\right)$, is record for $i=1,2, \ldots, m$, where $w_{i}=\{1,0\}$ is denoted to unit type, from line $A_{1}$ or line $A_{2}$, respectively. Then, at any step of the experiment, when the failure time $\left(T_{i}, w_{i}\right)$ is observed, $R_{i}$ survival units are randomly removed from the experiment. The experiment is terminated when $\min \left(\tau, T_{m}\right)$ is recorded and the random sample, say $\mathbf{T}=\left\{\left(T_{1}, w_{1}\right),\left(T_{2}, w_{2}\right), \ldots,\left(T_{J}, w_{J}\right)\right\}, 1 \leq J \leq m$, is obtained. However, if we consider the jointly progressive hybrid typeII censoring scheme, the experiment is terminated when the $\max \left(\tau, T_{m}\right)$ is observed and the random sample, say $\mathbf{T}=\left\{\left(T_{1}, w_{1}\right),\left(T_{2}, w_{2}\right), \ldots,\left(T_{J}, w_{J}\right)\right\}, \quad m \leq J \leq n, \quad$ is obtained.

Balanced joint progressive type-II censoring is introduced early by [13] which is easier to handle than the joint progressive type-II censoring scheme. Different statistical properties of two exponential distributions under this scheme are discussed by [13]. This study is extended for Weibull lifetime distribution by [14]. Recently, inferences of Weibull parameters are underbalance two-sample type-II progressive censoring scheme [15]. In a balanced joint progressive type-II censoring scheme, we suppose two lines of production, $A_{1}$ and $A_{2}$, have the same kind of products under the same facility and the sample of size $\left(\kappa_{1}+\kappa_{2}\right)$ with size $\kappa_{1}$ from $A_{1}$ and $\kappa_{2}$ from $A_{2}$ are put under life testing. Also, the prior integer $m$ is denoted to the number of failed units, and censoring scheme $\mathbf{R}=\left\{R_{1}, R_{2}, \ldots, R_{m-1}\right\}$ is determined to satisfy $m+\sum_{i=1}^{m-1} R_{i}<\min \left(\kappa_{1}, \kappa_{2}\right)$. If the first failure $T_{1}$ is observed from the line $A_{1}$, then $R_{1}$ and $\left(R_{1}+1\right)$ survival units are removed from the sample $\kappa_{1}$ and $\kappa_{2}$, respectively. Also, if the second failure $T_{2}$ is observed from the line $A_{2}$, then $\left(R_{2}+1\right)$ and $R_{2}$ survival units are removed from the sample $\left(\kappa_{1}-1-R_{1}\right)$ and $\left(\kappa_{2}-1-R_{1}\right)$, respectively. Then, the test is continual until $m$ th failure is reached. If the $m$ th failure is from the line $A_{1}$, then the remaining $\left(\kappa_{1}-m-\sum_{i=1}^{m-1} R_{i}\right)$ and $\left(\kappa_{2}-(m-1)-\sum_{i=1}^{m-1} R_{i}\right)$ survival units drown from the test. And, if the $m$ th failure is from the line $A_{2}$, then the remaining $\left(\kappa_{1}-(m-1)-\sum_{i=1}^{m-1} R_{i}\right)$ and $\left(\kappa_{2}-m-\sum_{i=1}^{m-1} R_{i}\right)$ survival units drown from the test. Then, the observed balanced joint progressive type-II censoring sample is given by $\underline{\mathbf{t}}=\left\{\left(t_{1}, w_{1}\right),\left(t_{2}, w_{2}\right), \ldots,\left(t_{m}, w_{m}\right)\right\}$.

This paper aims to use the progressive hybrid type-I censoring scheme under a balanced joint technique to present the BJPHCS. The BJPHCS is analytically easier to handle than another joint censoring scheme. Also, the properties of the estimators under BJPHCS can be stated more explicitly. Under this scheme, we construct the likelihood function for the model parameters. The lifetime information obtained from Lomax lifetime distribution under BJPHCS is used to present the two Lomax life distributions' statistical inference. Also, the asymptotic confidence interval under the normality distribution of the estimates is constructed. The Bayes estimators of unknown model parameters with the corresponding credible intervals are developed. Different estimators are discussed and compared through the Monte Carlo simulation study and illustrated through a numerical example based on BJPHCS.

The paper is constructed with the following sections. The concepts and model of the formulation are built in Section 2. The model parameters are estimated with maximum likelihood estimation in Section 3. The Bayes estimation and the corresponding credible intervals are derived in Section 4. Numerical discussion in the form of illustrative example and Monto Carlo simulation studies are reported in Section 5. Some comments are built to discuss the numerical results in Section 6.

\section{The Model}

Suppose the product comes from different two lines $A_{1}$ and $A_{2}$ of production under the same conditions. And, let the sample of size $\left(\kappa_{1}+\kappa_{2}\right)$ be randomly selected with $\kappa_{1}$ drawn from the line $A_{1}$ and $\kappa_{2}$ drawn from the line $A_{2}$. Prior to the beginning of the experiment, the experimenter determines the number $m$ of failure units, the ideal test time $\tau$, and the nonnegative censoring scheme $\underline{\mathbf{R}}=\left\{R_{1}, R_{2}, \ldots, R_{m}\right\}$ to satisfy $m+\sum_{i=1}^{m-1} R_{i}<\min \left(\kappa_{1}, \kappa_{2}\right)$. When the experiment is beginning, the time to failure and its type are recorded, which means from line $A_{1}$ or line $A_{2}$. If the first failure $T_{1}$ is recorded from the line $A_{1}$, then $R_{1}$ and $\left(R_{1}+1\right)$ survival units are removed from the sample $\kappa_{1}$ and $\kappa_{2}$, respectively. Also, if the second failure $T_{2}$ is observed from the line $A_{2}$, then $\left(R_{2}+1\right)$ and $R_{2}$ survival units are removed from the sample $\left(\kappa_{1}-R_{1}-1\right)$ and $\left(\kappa_{2}-R_{1}-1\right)$, respectively. Then, the experiment is continual until the $\min \left(\tau, T_{m}\right)$ is observed. If $T_{m}<\tau$, the results are similar to balanced joint progressive type-II censoring [13], but, if $T_{m}>\tau$, the experiment is removed at $\tau$, where $T_{J}<\tau<T_{J+1}$. If the $J$ th failure is from the line $A_{1}$, then the remaining $\left(\kappa_{1}-J-\right.$ $\left.\sum_{i=1}^{J-1} R_{i}\right)$ and $\left(\kappa_{2}-(J-1)-\sum_{i=1}^{J-1} R_{i}\right)$ survival units drown from the test. And, if the $J$ th failure is from the line $A_{2}$, then the remaining $\left(\kappa_{1}-(J-1)-\sum_{i=1}^{J-1} R_{i}\right)$ and $\left(\kappa_{2}-J-\sum_{i=1}^{J-1} R_{i}\right)$ survival units drown from the test. Then, the observed BJPHC sample is given by $\underline{\mathbf{t}}=\left\{\left(t_{1}, w_{1}\right),\left(t_{2}\right.\right.$, $\left.\left.w_{2}\right), \ldots,\left(t_{J}, w_{J}\right)\right\}$. Figure 1 shows the plane of BJPHCS. Suppose $\kappa_{1}$ units have the identical independent distributed (i.i.d.) random lifetimes $X_{1}, X_{2}, \ldots, X_{\kappa_{1}}$ and $\kappa_{2}$ units have the i.i.d. random lifetimes $Y_{1}, Y_{2}, \ldots, Y_{\kappa_{2}}$. The two samples are distributed with distribution with probability density functions (PDFs), and the cumulative distribution functions $(\mathrm{CDFs})$ are given by $f_{l}($.$) and F_{l}(),. l=1,2$. Then, the ordered sample $\left\{T_{1}, T_{2}, \ldots, T_{J}\right\}, 1 \leq J \leq m$, is formulated from the joint sample $\left\{X_{1}, X_{2}, \ldots, X_{J_{1}}, Y_{1}, Y_{2}, \ldots, Y_{J_{2}}\right\}$, where $J=J_{1}+J_{2}$ and $J_{1}$ is the number of units fails from the line $A_{1}$ and $J_{2}$ is the number of units fails from the line $A_{2}$. The observed BJPHCS censoring sample $\underline{\mathbf{t}}=\left\{\left(t_{1}, w_{1}\right),\left(t_{2}, w_{2}\right), \ldots,\left(t_{J}, w_{J}\right)\right\}$ in which $w_{i}$ is 1 or 0 values and depends on line $A_{1}$ or $A_{2}$, respectively, and $J_{1}=$ $\sum_{i=1}^{J} w_{i}$ and $J_{2}=\sum_{i=1}^{J}\left(1-w_{i}\right)$.

Hence, under observed sample $\underline{\mathbf{t}}=\left\{\left(t_{1}, w_{1}\right),\left(t_{2}, w_{2}\right), \ldots,\left(t_{J}, w_{J}\right)\right\}$, the likelihood function is given by 


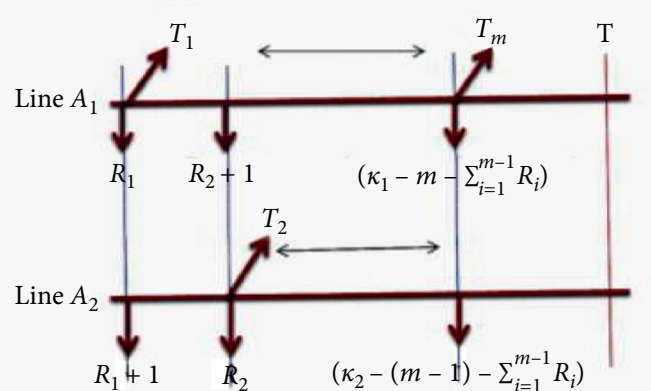

(a)

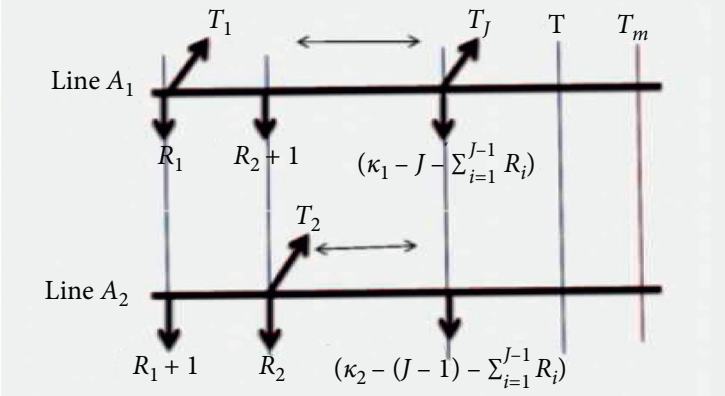

(b)

FIgURe 1: Schematic diagram of BJPHCS.

$$
f_{1,2, \ldots, J}(\underline{\mathbf{t}} \mid \Phi) \propto\left(\prod_{i=1}^{J}\left[h_{1}\left(t_{i}\right)\right]^{w_{i}}\left[h_{2}\left(t_{i}\right)\right]^{1-w_{i}}\left[S_{1}\left(t_{i}\right) S_{2}\left(t_{i}\right)\right]^{R_{i}+1}\right) \times\left(S_{1}\left(\tau^{*}\right)\right)^{\kappa_{1}-J-}\left(\sum_{i=1}^{J} R_{i}\right) S_{2}\left(\tau^{*}\right)^{\kappa_{2}-J-}\left(\sum_{i=1}^{J} R_{i}\right)
$$

where $\tau^{*}=\min \left(\tau, T_{m}\right), \Phi$ is the parameters vector, and $S_{j}($. and $h_{j}(),. j=1,2$, are denoted as reliability and hazard rate functions, respectively. Different lines $A_{1}$ and $A_{2}$ of production with units have PDFs, and CDFs follow Lomax lifetime distribution defined with PDFs and given by

$$
f_{l}(t)=\frac{\alpha_{l}}{\beta_{l}}\left(1+\frac{t}{\beta_{l}}\right)^{-\left(\alpha_{l}+1\right)}, \quad t>0, \alpha_{l}, \beta_{l}>0, j=1,2,
$$

and $\mathrm{CDFs}$ are given by

$$
F_{l}(t)=1-\left(1+\frac{t}{\beta_{l}}\right)^{-\alpha_{l}} .
$$

The reliability and hazard rate functions of Lomax lifetime distributions is also given by

$$
L\left(\alpha_{1}, \alpha_{2}, \beta_{1}, \beta_{2} \mid \underline{\mathbf{t}}\right) \propto \alpha_{1}^{J_{1}} \alpha_{2}^{J_{2}} \prod_{i=1}^{J}\left\{\left(\beta_{1}+t_{i}\right)^{-w_{i}}\left(\beta_{2}+t_{i}\right)^{-\left(1-w_{i}\right)}\left(1+\frac{t_{i}}{\beta_{1}}\right)^{-\alpha_{1}\left(R_{i}+1\right)}\left(1+\frac{t_{i}}{\beta_{2}}\right)^{-\alpha_{2}\left(R_{i}+1\right)}\right\}\left(1+\frac{\tau^{*}}{\beta_{1}}\right)^{-\alpha_{1} K_{1}}\left(1+\frac{\tau^{*}}{\beta_{2}}\right)^{-\alpha_{2} K_{2}},
$$

where

$$
K_{i}=\kappa_{i}-J-\sum_{i=1}^{J} R_{i}, \quad i=1,2 .
$$

The joint likelihood function is defined by (6) after the natural logarithms are reduced to

$$
\begin{aligned}
& S_{l}(t)=\left(1+\frac{t}{\beta_{l}}\right)^{-\alpha_{l}}, \\
& h_{l}(t)=\frac{\alpha_{l}}{\beta_{l}+t} .
\end{aligned}
$$

The Lomax lifetime distribution has been introduced early in [16] and more information about this distribution has been introduced in [17]. Lomax lifetime distribution is heavy-tailed; this propriety makes a more suitable distribution than exponential, gamma, and Weibull lifetime distribution. The failure time of Lomax lifetime distribution under different risks is studied by [18] and recently by [19].

\section{Maximum Likelihood Estimation}

For the given joint sample $\mathbf{t}=\left\{\left(t_{1}, w_{1}\right),\left(t_{2}, w_{2}\right), \ldots,\left(t_{J}, w_{J}\right)\right\}$ and Lomax distribution given by (2)-(5), the joint likelihood function (1) is reduced to 


$$
\begin{aligned}
\ell\left(\alpha_{1}, \alpha_{2}, \beta_{1}, \beta_{2} \mid \underline{\mathbf{t}}\right)= & J_{1} \log \alpha_{1}+J_{2} \log \alpha_{2}-\sum_{i=1}^{J} w_{i} \log \left(\beta_{1}+t_{i}\right)-\sum_{i=1}^{J}\left(1-w_{i}\right) \\
& \times \log \left(\beta_{2}+t_{i}\right)-\sum_{i=1}^{J}\left(R_{i}+1\right)\left(\alpha_{1} \log \left(1+\frac{t_{i}}{\beta_{1}}\right)+\alpha_{2} \log \left(1+\frac{t_{i}}{\beta_{2}}\right)\right)-\alpha_{1} K_{1} \log \left(1+\frac{\tau^{*}}{\beta_{1}}\right)-\alpha_{2} K_{2} \log \left(1+\frac{\tau^{*}}{\beta_{2}}\right) .
\end{aligned}
$$

The likelihood equations can be obtained from logarithm (8) by taking the first partial derivative with respect to model parameters, and hence, the point and interval estimate can be formulated as follows.

3.1. MLEs. Let $\Phi=\left\{\alpha_{1}, \alpha_{2}, \beta_{1}, \beta_{2}\right\}$ be the model parameters vector; then, the zero values of derivative of log-likelihood function are described by,

$$
\frac{\partial \ell\left(\alpha_{1}, \alpha_{2}, \beta_{1}, \beta_{2} \mid \underline{t}\right)}{\partial \alpha_{j}}=0, \quad j=1,2,
$$

and described by,

$$
\begin{aligned}
& \alpha_{1}=\frac{J_{1}}{\sum_{i=1}^{J}\left(R_{i}+1\right) \log \left(1+\left(t_{i} / \beta_{1}\right)\right)+K_{1} \log \left(1+\left(\tau^{*} / \beta_{1}\right)\right)}, \\
& \alpha_{2}=\frac{J_{2}}{\sum_{i=1}^{J}\left(R_{i}+1\right) \log \left(1+\left(t_{i} / \beta_{2}\right)\right)+K_{2} \log \left(1+\left(\tau^{*} / \beta_{2}\right)\right)} .
\end{aligned}
$$

Also,

$$
\frac{\partial \ell\left(\alpha_{1}, \alpha_{2}, \beta_{1}, \beta_{2} \mid \underline{\mathbf{t}}\right)}{\partial \beta_{j}}=0, \quad j=1,2,
$$

which is described by,

$$
\begin{gathered}
-\sum_{i=1}^{J} \frac{w_{i}}{\beta_{1}+t_{i}}+\left(\frac{\alpha_{1}}{\beta_{1}}\right)\left(\sum_{i=1}^{J} \frac{\left(R_{i}+1\right) t_{i}}{\beta_{1}+t_{i}}+\frac{K_{1} \tau^{*}}{\left(\beta_{1}+\tau\right)}\right)=0, \\
-\sum_{i=1}^{J} \frac{\left(1-w_{i}\right)}{\beta_{2}+t_{i}}+\left(\frac{\alpha_{2}}{\beta_{2}}\right)\left(\sum_{i=1}^{J} \frac{\left(R_{i}+1\right) t_{i}}{\beta_{2}+t_{i}}+\frac{K_{2} \tau^{*}}{\left(\beta_{2}+\tau\right)}\right)=0 .
\end{gathered}
$$

Equations (13) and (14) after replacing $\alpha_{i}, i=1,2$, from (10) and (11), are reduced to

$$
\begin{aligned}
& -\sum_{i=1}^{J} \frac{w_{i}}{\beta_{1}+t_{i}}+\frac{\left(J_{1} / \beta_{1}\right)\left(\sum_{i=1}^{J}\left(\left(R_{i}+1\right) t_{i} / \beta_{1}+t_{i}\right)+\left(K_{1} \tau^{*} /\left(\beta_{1}+\tau\right)\right)\right)}{\sum_{i=1}^{J}\left(R_{i}+1\right) \log \left(1+\left(t_{i} / \beta_{1}\right)\right)+K_{1} \log \left(1+\left(\tau^{*} / \beta_{1}\right)\right)}=0 \\
& -\sum_{i=1}^{J} \frac{\left(1-w_{i}\right)}{\beta_{2}+t_{i}}+\frac{\left(J / \beta_{2}\right)\left(\sum_{i=1}^{J}\left(\left(R_{i}+1\right) t_{i} / \beta_{2}+t_{i}\right)+\left(K_{2} \tau^{*} /\left(\beta_{2}+\tau\right)\right)\right)}{\sum_{i=1}^{J}\left(R_{i}+1\right) \log \left(1+\left(t_{i} / \beta_{2}\right)\right)+K_{2} \log \left(1+\left(\tau^{*} / \beta_{2}\right)\right)}=0
\end{aligned}
$$

The nonlinear equations (15) and (16) are simply solved with Newton-Raphson iteration to obtain $\widehat{\beta}_{1}$ and $\widehat{\beta}_{2}$. Then, the estimates $\widehat{\alpha}_{1}$ and $\widehat{\alpha}_{2}$ are obtained from (10) and (11).

Remark 1. For each of the parameters, $\alpha_{i}$ and $\beta_{i}, i=1,2$, do not exist for the case $J_{1}=0$ as well as $J_{2}=0$. Also, if $J_{1}=1$ as well as $J_{2}=1$, the parameters $\alpha_{i}$ and $\beta_{i}$ are not estimable. Hence, the MLEs in (10), (11), (15), and (16) are only conditional MLEs, conditioned on $1<J_{1}$ and $1<J_{2}$. Hence, the properties of the MLEs are discussed only as conditional on $1<J_{1}$ and $1<J_{2}$ [20]. Also, the exact distributions for estimators $\widehat{\Phi}=\left\{\widehat{\alpha}_{1}, \widehat{\alpha}_{2}, \widehat{\beta}_{1}, \widehat{\beta}_{2}\right\}$ are difficult to obtain [21].

3.2. Approximate Confidence Interval. Fisher information matrix is defined as the minus expectation of the second derivative of log-likelihood function with respect to the model parameters' vectors $\Sigma=-E\left(\partial^{2} \ell\left(\alpha_{1}, \alpha_{2}, \beta_{1}\right.\right.$, $\left.\left.\beta_{2} \mid \mathbf{t}\right) / \partial \Phi_{i} \partial \Phi_{j}\right), i, j=1,2,3,4$. For the cases of a large sample, we can use the approximate information matrix, which is obtained under maximum likelihood estimates and denoted by $\Sigma_{0}\left(\widehat{\alpha}_{1}, \widehat{\alpha}_{2}, \widehat{\beta}_{1}, \widehat{\beta}_{2}\right)$ which is applied as alternative of Fisher information matrix. The approximate confidence intervals for model parameters $\Phi=\left\{\alpha_{1}, \alpha_{2}, \beta_{1}, \beta_{2}\right\}$ are dependent on the asymptotic normality distribution of estimates $\widehat{\alpha}_{1}, \widehat{\alpha}_{2}, \widehat{\beta}_{1}$, and $\widehat{\beta}_{2}$ with mean $\left\{\alpha_{1}, \alpha_{2}, \beta_{1}, \beta_{2}\right\}$ and variance covariance matrix $\Sigma_{0}^{-1}\left(\widehat{\alpha}_{1}, \widehat{\alpha}_{2}, \widehat{\beta}_{1}, \widehat{\beta}_{2}\right)$; then, we can say that $\widehat{\Phi}=\left(\widehat{\alpha}_{1}, \widehat{\alpha}_{2}, \widehat{\beta}_{1}, \widehat{\beta}_{2}\right)$ is distributed as normal distribution as follows:

$$
\widehat{\Phi} \sim N\left(\left(\alpha_{1}, \alpha_{2}, \beta_{1}, \beta_{2}\right), \Sigma_{0}^{-1}\left(\widehat{\alpha}_{1}, \widehat{\alpha}_{2}, \widehat{\beta}_{1}, \widehat{\beta}_{2}\right)\right),
$$

and $\Sigma_{0}^{-1}\left(\widehat{\alpha}_{1}, \widehat{\alpha}_{2}, \widehat{\beta}_{1}, \widehat{\beta}_{2}\right)$ is given by 


$$
\Sigma_{0}^{-1}\left(\widehat{\alpha}_{1}, \widehat{\alpha}_{2}, \widehat{\beta}_{1}, \widehat{\beta}_{2}\right)=\left[\begin{array}{l}
-\frac{\partial^{2} \ell\left(\alpha_{1}, \alpha_{2}, \beta_{1}, \beta_{2} \mid \underline{\mathbf{t}}\right)}{\partial \alpha_{1}^{2}}-\frac{\partial^{2} \ell\left(\alpha_{1}, \alpha_{2}, \beta_{1}, \beta_{2} \mid \underline{\mathbf{t}}\right)}{\partial \alpha_{1} \partial \alpha_{2}}-\frac{\partial^{2} \ell\left(\alpha_{1}, \alpha_{2}, \beta_{1}, \beta_{2} \mid \underline{\mathbf{t}}\right)}{\partial \alpha_{1} \partial \beta_{1}}-\frac{\partial^{2} \ell\left(\alpha_{1}, \alpha_{2}, \beta_{1}, \beta_{2} \mid \underline{\mathbf{t}}\right)}{\partial \alpha_{1} \partial \beta_{2}} \\
-\frac{\partial^{2} \ell\left(\alpha_{1}, \alpha_{2}, \beta_{1}, \beta_{2} \mid \underline{\mathbf{t}}\right)}{\partial \alpha_{2} \partial \alpha_{1}}-\frac{\partial^{2} \ell\left(\alpha_{1}, \alpha_{2}, \beta_{1}, \beta_{2} \mid \underline{\mathbf{t}}\right)}{\partial \alpha_{2}^{2}}-\frac{\partial^{2} \ell\left(\alpha_{1}, \alpha_{2}, \beta_{1}, \beta_{2} \mid \underline{\mathbf{t}}\right)}{\partial \alpha_{2} \partial \beta_{1}}-\frac{\partial^{2} \ell\left(\alpha_{1}, \alpha_{2}, \beta_{1}, \beta_{2} \mid \underline{\mathbf{t}}\right)}{\partial \alpha_{2} \partial \beta_{2}} \\
-\frac{\partial^{2} \ell\left(\alpha_{1}, \alpha_{2}, \beta_{1}, \beta_{2} \mid \underline{\mathbf{t}}\right)}{\partial \beta_{1} \partial \alpha_{1}}-\frac{\partial^{2} \ell\left(\alpha_{1}, \alpha_{2}, \beta_{1}, \beta_{2} \mid \underline{\mathbf{t}}\right)}{\partial \beta_{1} \partial \alpha_{2}}-\frac{\partial^{2} \ell\left(\alpha_{1}, \alpha_{2}, \beta_{1}, \beta_{2} \mid \underline{\mathbf{t}}\right)}{\partial \beta_{1}^{2}}-\frac{\partial^{2} \ell\left(\alpha_{1}, \alpha_{2}, \beta_{1}, \beta_{2} \mid \underline{\mathbf{t}}\right)}{\partial \beta_{1} \partial \beta_{2}} \\
-\frac{\partial^{2} \ell\left(\alpha_{1}, \alpha_{2}, \beta_{1}, \beta_{2} \mid \underline{\mathbf{t}}\right)}{\partial \beta_{2} \partial \alpha_{1}}-\frac{\partial^{2} \ell\left(\alpha_{1}, \alpha_{2}, \beta_{1}, \beta_{2} \mid \underline{\mathbf{t}}\right)}{\partial \beta_{2} \partial \alpha_{2}}-\frac{\partial^{2} \ell\left(\alpha_{1}, \alpha_{2}, \beta_{1}, \beta_{2} \mid \underline{\mathbf{t}}\right)}{\partial \beta_{2} \partial \beta}-\frac{\partial^{2} \ell\left(\alpha_{1}, \alpha_{2}, \beta_{1}, \beta_{2} \mid \underline{\mathbf{t}}\right)}{\partial \beta_{2}^{2}}
\end{array}\right],
$$

at $\widehat{\Phi}=\left(\widehat{\alpha}_{1}, \widehat{\beta}_{1}, \widehat{\alpha}_{2}, \widehat{\beta}_{2}\right)$.

Then, at confidence level $\gamma, 100(1-\gamma) \%$ approximate confidence intervals of $\alpha_{1}, \alpha_{2}, \beta_{1}$, and $\beta_{2}$, respectively, are given by

$$
\left\{\begin{array}{l}
\widehat{\alpha}_{i} \mp z_{(\gamma / 2)} \sqrt{e_{i i}} \\
\widehat{\beta}_{i} \mp z_{(\gamma / 2)} \sqrt{e_{i+2 i+2}}
\end{array}, \quad i=1,2,\right.
$$

where the values $e_{11}, e_{22}, e_{33}$, and $e_{44}$ are the diagonal of matrix $\Sigma_{0}^{-1}$ with standard normal value $z_{\gamma / 2}$ with right-tail probability $(\gamma / 2)$. The second derivative of log-likelihood function with respect to the model parameters vector $\Phi=$ $\left\{\alpha_{1}, \alpha_{2}, \beta_{1}, \beta_{2}\right\}$ are given by

$$
\begin{aligned}
& \frac{\partial^{2} \ell\left(\alpha_{1}, \alpha_{2}, \beta_{1}, \beta_{2} \mid \underline{\mathbf{t}}\right)}{\partial \alpha_{i}^{2}}=\frac{-J_{i}}{\alpha_{i}^{2}}, \quad i=1,2, \\
& \frac{\partial^{2} \ell\left(\alpha_{1}, \alpha_{2}, \beta_{1}, \beta_{2} \mid \underline{\mathbf{t}}\right)}{\partial \beta_{1}^{2}}=\sum_{i=1}^{J} \frac{w_{i}}{\left(\beta_{1}+t_{i}\right)^{2}}-\left(\frac{\alpha_{1}}{\beta_{1}^{2}}\right)\left\{\sum_{i=1}^{J} \frac{\left(R_{i}+1\right) t_{i}\left(2 \beta_{1}+t_{i}\right)}{\left(\beta_{1}+t_{i}\right)^{2}}-\frac{K_{1} \tau\left(2 \beta_{1}+\tau^{*}\right)}{\left(\beta_{1}+\tau\right)^{2}}\right\} \\
& \frac{\partial^{2} \ell\left(\alpha_{1}, \alpha_{2}, \beta_{1}, \beta_{2} \mid \underline{\mathbf{t}}\right)}{\partial \beta_{2}^{2}}=\sum_{i=1}^{J} \frac{\left(1-w_{i}\right)}{\left(\beta_{2}+t_{i}\right)^{2}}-\left(\frac{\alpha_{2}}{\beta_{2}^{2}}\right)\left\{\sum_{i=1}^{J} \frac{\left(R_{i}+1\right) t_{i}\left(2 \beta_{2}+t_{i}\right)}{\left(\beta_{2}+t_{i}\right)^{2}}-\frac{K_{2} \tau\left(2 \beta_{2}+\tau\right)}{\left(\beta_{2}+\tau\right)^{2}}\right\} \\
& \frac{\partial^{22} \ell\left(\alpha_{1}, \alpha_{2}, \beta_{1}, \beta_{2} \mid \underline{t}\right)}{\partial \alpha_{1} \partial \beta_{1}}=\frac{\partial^{22} \ell\left(\alpha_{1}, \alpha_{2}, \beta_{1}, \beta_{2} \mid \underline{t}\right)}{\partial \beta_{1} \partial \alpha_{1}}=\frac{1}{\beta_{1}}\left(\sum_{i=1}^{J} \frac{\left(R_{i}+1\right) t_{i}}{\beta_{1}+t_{i}}+\frac{K_{1} \tau^{*}}{\left(\beta_{1}+\tau\right)}\right) \\
& \frac{\partial^{2} \ell\left(\alpha_{1}, \alpha_{2}, \beta_{1}, \beta_{2} \mid \underline{\mathbf{t}}\right)}{\partial \alpha_{2} \partial \beta_{2}}=\frac{\partial^{2} \ell\left(\alpha_{1}, \alpha_{2}, \beta_{1}, \beta_{2} \mid \underline{\mathbf{t}}\right)}{\partial \beta_{2} \partial \alpha_{2}}=\frac{1}{\beta_{2}}\left(\sum_{i=1}^{J} \frac{\left(R_{i}+1\right) t_{i}}{\beta_{2}+t_{i}}+\frac{K_{2} \tau^{*}}{\left(\beta_{2}+\tau\right)}\right) \\
& \frac{\partial^{2} \ell\left(\alpha_{1}, \alpha_{2}, \beta_{1}, \beta_{2} \mid \underline{\mathbf{t}}\right)}{\partial \alpha_{1} \partial \beta_{2}}=\frac{\partial^{2} \ell\left(\alpha_{1}, \alpha_{2}, \beta_{1}, \beta_{2} \mid \underline{\mathbf{t}}\right)}{\partial \beta_{2} \partial \alpha_{1}}=\frac{\partial^{2} \ell\left(\alpha_{1}, \alpha_{2}, \beta_{1}, \beta_{2} \mid \underline{\mathbf{t}}\right)}{\partial \alpha_{1} \partial \alpha_{2}}= \\
& \frac{\partial^{2} \ell\left(\alpha_{1}, \alpha_{2}, \beta_{1}, \beta_{2} \mid \underline{\mathbf{t}}\right)}{\partial \alpha_{2} \partial \alpha_{1}}=\frac{\partial^{2} \ell\left(\alpha_{1}, \alpha_{2}, \beta_{1}, \beta_{2} \mid \underline{\mathbf{t}}\right)}{\partial \alpha_{2} \partial \beta_{1}}=\frac{\partial^{2} \ell\left(\alpha_{1}, \alpha_{2}, \beta_{1}, \beta_{2} \mid \underline{\mathbf{t}}\right)}{\partial \beta_{1} \partial \alpha_{2}}= \\
& \frac{\partial^{2} \ell\left(\alpha_{1}, \alpha_{2}, \beta_{1}, \beta_{2} \mid \underline{\mathbf{t}}\right)}{\partial \beta_{1} \partial \beta_{2}}=\frac{\partial^{2} \ell\left(\alpha_{1}, \alpha_{2}, \beta_{1}, \beta_{2} \mid \underline{\mathbf{t}}\right)}{\partial \beta_{2} \partial \beta_{1}}=0 .
\end{aligned}
$$

\section{Bayes Estimation}

The Bayesian approach for parameters' estimation depends on the prior information available about the parameters and the data presented. Gamma distribution characterized by different shapes depends on its parameters; this property has marked it to be a more suitable distribution in other cases. So, we consider the independent gamma prior for distribution of model parameters as follows: 


$$
\Pi_{l}^{*}\left(\Phi_{l}\right)=\frac{b_{l}^{a_{l}}}{\Gamma\left(a_{l}\right)} \Phi_{l}^{a_{l}-1} \exp \left(-b_{l} \Phi_{l}\right), \quad l=1,2,3,4,
$$

where $\Phi$ presents the parameters' vector. The joint prior density is given by

$$
\Pi^{*}(\Phi)=\prod_{l=1}^{4} \Pi_{l}^{*}\left(\Phi_{l}\right)
$$

Also, after obtaining the data in the form of balanced joint progressive hybrid type-I censoring data which formed by likelihood function, then, we construct all information about the parameters by posterior probability density defined by

$$
\Pi(\Phi \mid \underline{t})=Q \Pi^{*}(\Phi) L(\Phi \mid \underline{t})
$$

where $Q=\int_{\Phi} \Pi^{*}(\Phi) L(\Phi \mid \underline{t}) \mathrm{d} \Phi$. Then, the Bayes estimators for the function $\zeta(\Phi)$ under squared error loss function (SEL) is given by

$$
\widehat{\Phi}_{B}=Q \int_{\Phi} \zeta(\Phi) \Pi^{*}(\Phi) L(\Phi \mid \underline{t}) \mathrm{d} \Phi .
$$

Hence, the Bayes estimators have two integrables, which need approximation methods such as numerical integration or Lindley approximation. The more general case is applied in different Bayesian computation areas called as Markov chain Monto Carlo (MCMC) method.

MCMC approach: to adopt the Bayesian approach under the MCMC method, we obtain the conditional distributions of the posterior distribution, which is given by

$$
\Pi\left(\alpha_{1}, \alpha_{2}, \beta_{1}, \beta_{2} \mid \underline{\mathbf{t}}\right) \propto \exp \left\{\begin{array}{c}
-b_{1} \alpha_{1}-b_{2} \alpha_{2}-b_{3} \beta_{1}-b_{4} \beta_{2}-\sum_{i=1}^{J} w_{i} \log \left(\beta_{1}+t_{i}\right)-\sum_{i=1}^{J}\left(1-w_{i}\right) \\
\times \log \left(\beta_{2}+t_{i}\right)-\sum_{i=1}^{J}\left(R_{i}+1\right)\left(\alpha_{1} \log \left(1+\frac{t_{i}}{\beta_{1}}\right)+\alpha_{2} \log \left(1+\frac{t_{i}}{\beta_{2}}\right)\right)-\alpha_{1} K_{1} \\
\times \log \left(1+\frac{\tau^{*}}{\beta_{1}}\right)-\alpha_{2} K_{2} \log \left(1+\frac{\tau^{*}}{\beta_{2}}\right)
\end{array}\right\}
$$

The full conditional distributions of the parameters are given by

$$
\begin{gathered}
\Pi\left(\alpha_{1} \mid \alpha_{2}, \beta_{1}, \beta_{2} \underline{\mathbf{t}}\right) \propto \alpha_{1}^{J_{1}+a_{1}-1} \exp \left\{-\alpha_{1}\left(b_{1}+\sum_{i=1}^{J}\left(R_{i}+1\right) \log \left(1+\frac{t_{i}}{\beta_{1}}\right)+K_{1} \log \left(1+\frac{\tau^{*}}{\beta_{1}}\right)\right)\right\} \\
\Pi\left(\alpha_{2} \mid \alpha_{1}, \beta_{1}, \beta_{2}, \underline{\mathbf{t}}\right) \propto \alpha_{1}^{J_{2}+a_{2}-1} \exp \left\{-\alpha_{2}\left(b_{2}+\sum_{i=1}^{J}\left(R_{i}+1\right) \log \left(1+\frac{t_{i}}{\beta_{2}}\right)+K_{2} \log \left(1+\frac{\tau^{*}}{\beta_{2}}\right)\right)\right\} \\
\Pi\left(\beta_{1} \mid \alpha_{1}, \alpha_{2}, \beta_{2}, \underline{\mathbf{t}}\right) \propto \beta_{1}^{a_{3}-1} \exp \left\{-b_{3} \beta_{1}-\sum_{i=1}^{J} w_{i} \log \left(\beta_{1}+t_{i}\right)-\sum_{i=1}^{J}\left(R_{i}+1\right) \alpha_{1} \times \log \left(1+\frac{t_{i}}{\beta_{1}}\right)-\alpha_{1} K_{1} \log \left(1+\frac{\tau^{*}}{\beta_{1}}\right)\right\} \\
\Pi\left(\beta_{2} \mid \alpha_{1}, \alpha_{2}, \beta_{1}, \underline{\mathbf{t}}\right) \propto \beta_{2}^{a_{4}-1} \exp \left\{-b_{4} \beta_{2}-\sum_{i=1}^{J}\left(1-w_{i}\right) \log \left(\beta_{2}+t_{i}\right)-\alpha_{2} \sum_{i=1}^{J}\left(R_{i}+1\right) \times \log \left(1+\frac{t_{i}}{\beta_{2}}\right)-\alpha_{2} K_{2} \log \left(1+\frac{\tau^{*}}{\beta_{2}}\right)\right\} .
\end{gathered}
$$

Then, the posterior distribution is reduced to two conditional gamma functions and two distributions more similar to normal distribution. Then, the posterior distribution (25) and conditional posterior distributions (26) to 
TABLE 1: The generated balanced joint progressive type-I data.

\begin{tabular}{lccccccc}
\hline 0.03075 & 0.21431 & 0.23253 & 0.32229 & 0.39942 & 0.51726 & 0.80195 & 0.81130 \\
\hline 1 & 0 & 0 & 1 & 0 & 0 & 1 \\
0.83083 & 0.86332 & 1.29308 & 1.30155 & 1.46959 & 1.48689 & 1.66629 & 2.03257 \\
1 & 1 & 0 & 1 & 0 & 0 & 0 \\
2.16232 & 2.73166 & 2.75379 & 2.91897 & 4.33301 & 7.74818 & - \\
1 & 1 & 0 & 0 & 1 & 1 & - & - \\
\hline
\end{tabular}

Table 2: Point and 95\% confidence and credible intervals (ACIs and CIs) of MLEs Bayes estimates.

\begin{tabular}{|c|c|c|c|c|c|c|}
\hline Pa.s & $(.)_{\mathrm{ML}}$ & (.) $)_{\mathrm{BMCMC}}$ & 95\% ACIs & Length & $95 \% \mathrm{CIs}$ & Length \\
\hline$\alpha_{1}=1.2$ & 0.7215 & 0.8097 & $(0,1.9320)$ & 1.9320 & $(0.3969,1.4132)$ & 1.01626 \\
\hline$\beta_{1}=2.2$ & 2.8368 & 2.8453 & $(0,3.9947)$ & 3.9947 & $(1.4185,4.8630)$ & 3.4445 \\
\hline$\alpha_{2}=1.5$ & 0.4443 & 1.0680 & $(0,9.5860)$ & 9.5860 & $(0.5572,1.7875)$ & 1.23033 \\
\hline$\beta_{2}=2.5$ & 1.2865 & 3.2739 & $(0,3.7570)$ & 3.7570 & $(1.8051,5.2599)$ & 3.45486 \\
\hline
\end{tabular}

(29) have shown that MCMC methods are more suitable with more general Metropolis-Hastings $(\mathrm{MH})$ under Gibbs algorithms, see [22] described as follows.

\subsection{MH under Gibbs algorithm}

Step 1: for given initial vectors $\Phi^{(0)}=\left(\widehat{\alpha}_{1}, \widehat{\beta}_{1}, \widehat{\alpha}_{2}, \widehat{\beta}_{2}\right)$, put $I=1$

Step 2: generate two values $\alpha_{1}^{(I)}$ and $\alpha_{2}^{(I)}$ from conditional gamma distribution given by (26) and (27), respectively

Step 3: with proposal normal distributions, generate two values $\beta_{1}^{(I)}$ and $\beta_{2}^{(I)}$ from the conditional distributions (28) and (29)

Step 4: construct the iteration vector $\Phi^{(I)}=\left(\alpha_{1}^{(I)}, \alpha_{2}^{(I)}, \beta_{1}^{(I)}, \beta_{2}^{(I)}\right)$

Step 5: change $I$ to be $I+1$

Step 6: steps (2) to (5) are followed to get iteration procedure to $M$ times

Step 7: to reach the stationary state, we need iteration number $M^{*}$ which is known by "burn-in;" then, the Bayes estimators is given by

$$
\widehat{\zeta}(\Phi)_{\mathrm{B}}=E_{\Pi \mid \underline{t}}(\zeta(\Phi) \mid \underline{t})=\frac{1}{M-M^{*}} \sum_{i=M^{*}+1}^{M} \zeta^{(i)}\left(\Phi^{(i)}\right)
$$

as well as the corresponding posterior variance of $\zeta(\Phi)$ also, which is given by

$$
V(\zeta(\Phi) \mid \underline{t})=\frac{1}{M-M^{*}} \sum_{i=M^{*}+1}^{M}\left(\zeta^{(i)}\left(\Phi^{(i)}\right)-\widehat{\zeta}(\Phi)_{\mathrm{B}}\right)^{2}
$$

where $\zeta(\Phi)$ is a function of the parameters vector may be any one of them.

Step 8: the obtaining empirical distribution of $\zeta(\Phi)$ is obtained by the MCMC iteration after arranging its values ascending; then, the corresponding $100(1-\gamma) \%$ credible interval of $\zeta(\Phi)$ is given by

$$
\left(\zeta_{(\gamma / 2)\left(M-M^{*}\right)}, \zeta_{(1-(\gamma / 2))\left(M-M^{*}\right)}\right) \text {. }
$$

\section{Numerical Computations}

5.1. Example. Discuss and illustrate the proposed methods in this paper, firstly, about the relation between choosing the prior information that related with true parameters' values. The true parameters are chosen to satisfy that $E\left(\Phi_{i}\right) \simeq\left(a_{i} / b_{i}\right)$, see [23]. Also, the parameters are randomly generated from the known prior distribution; then, we can choose a set of parameters values generated from gamma distribution with parameters $(a, b)=\{(2,2),(4,2),(3,2),(5$, $2)\}$ to be $\Phi=\{1.2,2.2,1.5,2.5\}$. For the random selection, the two samples of size $\left(\kappa_{1}, \kappa_{2}\right)=(40,40)$ and prior integers $m=25$, censoring scheme $\mathbf{R}=\left\{14,0^{(23)}, 1\right\}$, and the ideal test time $\tau=10$. Hence, the randomly generated BJPHC data concerning to the algorithms are given by [24]. The generated data $\left(t_{i}, w_{i}\right)$ under the last consideration is summarized in Table 1 . For the data given in Table 1, the point MLE and corresponding confidence interval are summarized in Table 2. For the Bayesian approach, we run the chain 11,000 with the first 1000 value as burn-in the point, and the interval estimate is summarized in Table 2. Markov chain is built with desired properties and determines how many steps are needed to converge to the stationary distribution. The standard empirical method to assess convergence is also discussed with the plot of the first 11,000 steps for the Gibbs sampler shown in Figures 2 to 5. Then, the figures show the quality of the MCMC method in the Bayes method.

5.2. Simulation Studies. This section has examined the best choice of sample size, censoring scheme, and ideal test time. This discussion is reported for the classical ML and Bayes estimators of Lomax lifetime distribution under a balanced joint progressive hybrid type-I censoring scheme. Hence, the developed theoretical results are compared and assessed 


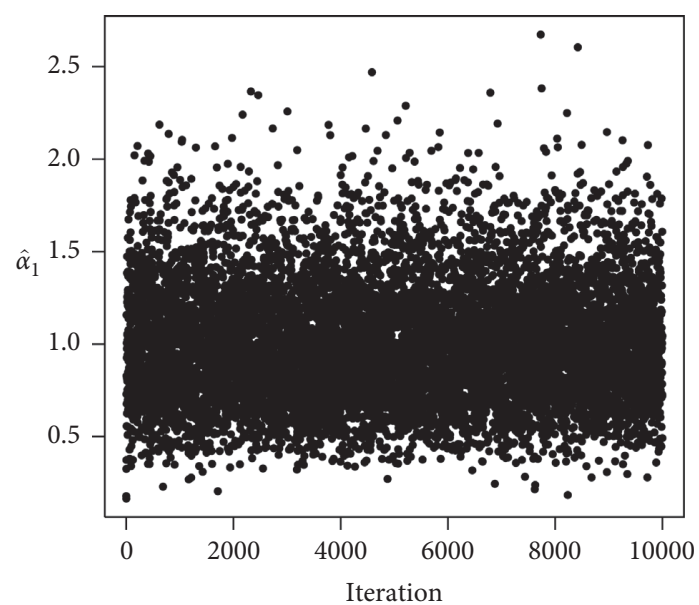

(a)

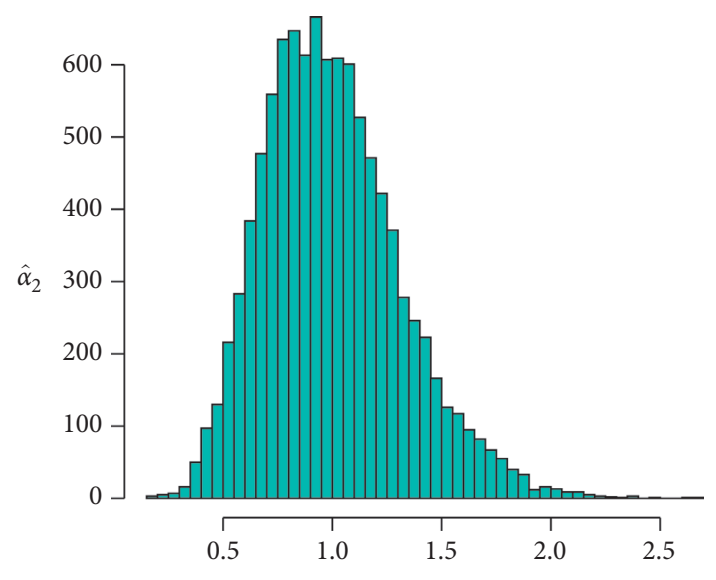

(b)

Figure 2: The simulation number and the corresponding histogram of $\alpha_{1}$ generated by the MCMC method.

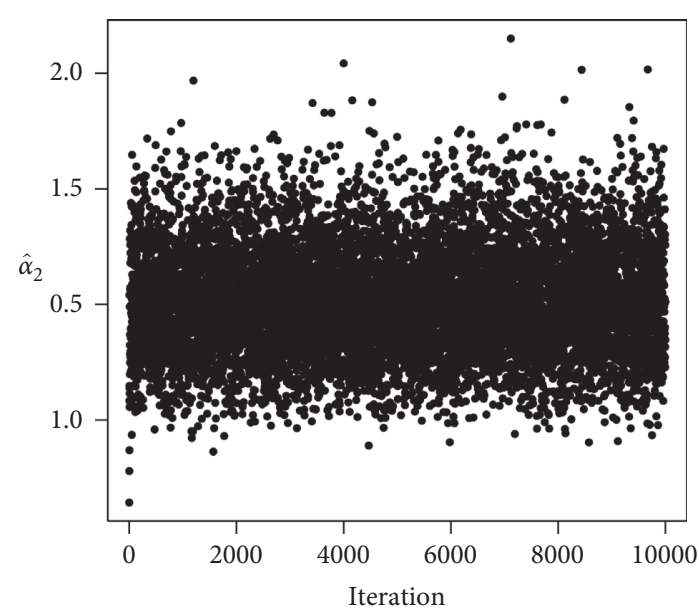

(a)

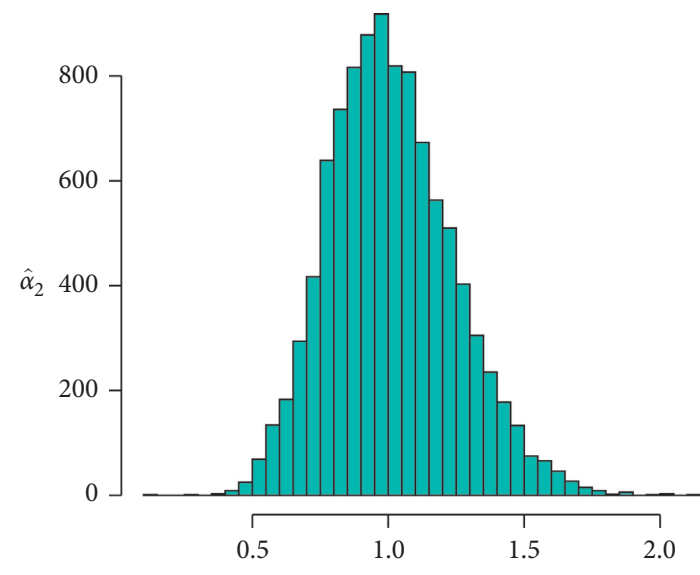

(b)

Figure 3: The simulation number and the corresponding histogram of $\alpha_{2}$ generated by the MCMC method.

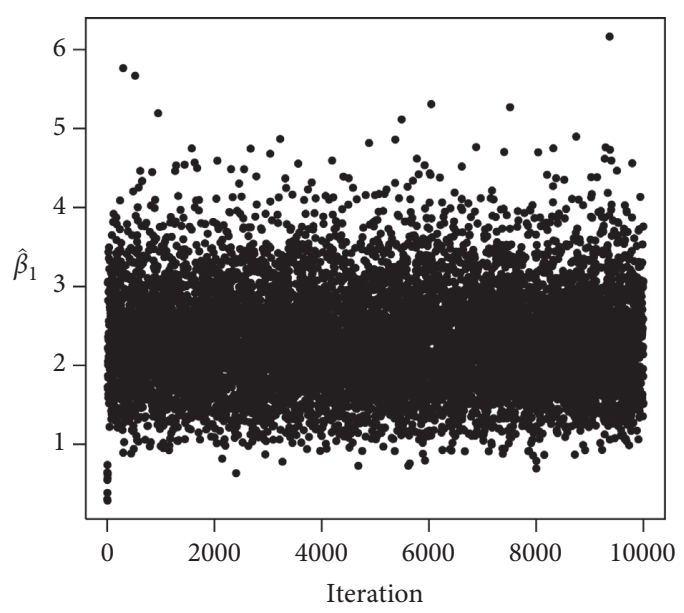

(a)

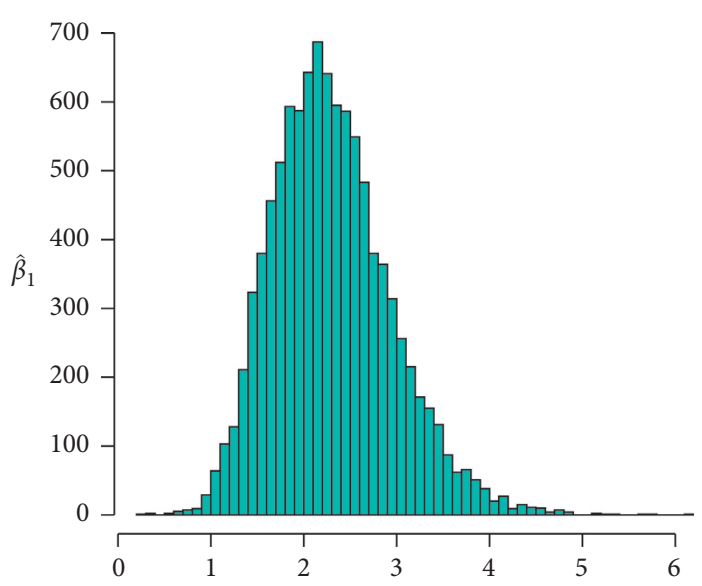

(b)

FIgURE 4: The simulation number and the corresponding histogram of $\beta_{1}$ generated by the MCMC method. 


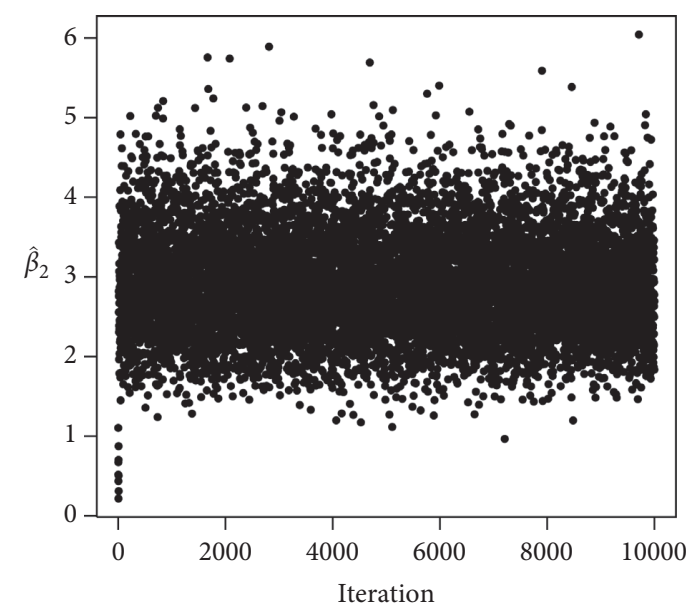

(a)

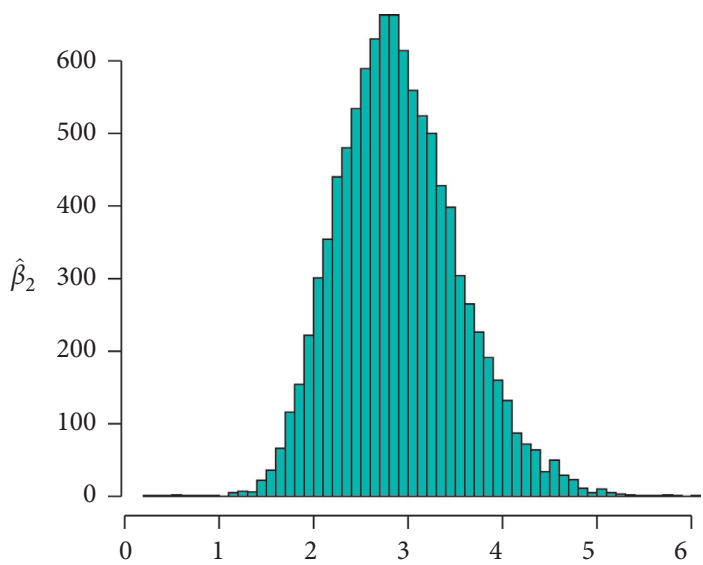

(b)

Figure 5: The simulation number and the corresponding histogram of $\beta_{2}$ generated by the MCMC method.

TABLE 3: MEs and $\sqrt{\mathrm{SV}}$ s of Lomax distributions with $\Phi=(3.0,1.0,2.0,1.5)$.

\begin{tabular}{|c|c|c|c|c|c|c|c|c|c|}
\hline \multirow{2}{*}{$\tau$} & \multirow{2}{*}{$\left(\kappa_{1}, \kappa_{2}\right)$} & \multirow{2}{*}{$(m,(\mathbf{R}))$} & \multirow{2}{*}{$\mathrm{Pa}$} & \multicolumn{2}{|c|}{ ML } & \multicolumn{2}{|c|}{$\mathrm{BMCMC}_{\text {prior }_{0}}$} & \multicolumn{2}{|c|}{$\mathrm{BMCMC}_{\text {prior }_{1}}$} \\
\hline & & & & AVs & MSEs & AVs & MSEs & AVs & MSEs \\
\hline \multirow{16}{*}{1.5} & \multirow{8}{*}{$(35,30)$} & \multirow{5}{*}{$\left(25,\left(5,0^{(24)}\right)\right)$} & $\alpha_{1}$ & 3.832 & 1.254 & 3.710 & 1.114 & 3.654 & 0.984 \\
\hline & & & $\alpha_{2}$ & 1.421 & 0.426 & 1.332 & 0.420 & 1.340 & 0.325 \\
\hline & & & $\beta_{1}$ & 2.315 & 0.754 & 2.374 & 0.701 & 2.214 & 0.654 \\
\hline & & & $\beta_{2}$ & 1.985 & 0.513 & 1.854 & 0.500 & 1.852 & 0.452 \\
\hline & & & $\alpha_{1}$ & 3.784 & 1.124 & 3.725 & 1.012 & 3.611 & 0.897 \\
\hline & & \multirow{4}{*}{$\left(25,\left(0^{(10)}, 5,0^{(10)}\right)\right)$} & $\alpha_{2}$ & 1.389 & 0.400 & 1.325 & 0.387 & 1.351 & 0.301 \\
\hline & & & $\beta_{1}$ & 2.301 & 0.715 & 2.312 & 0.698 & 2.223 & 0.578 \\
\hline & & & $\beta_{2}$ & 1.852 & 0.480 & 1.811 & 0.471 & 1.777 & 0.399 \\
\hline & \multirow{8}{*}{$(45,45)$} & & $\alpha_{1}$ & 3.452 & 0.985 & 3.411 & 0.979 & 3.412 & 0.745 \\
\hline & & \multirow{4}{*}{$\left(30,\left(15,0^{(29)}\right)\right)$} & $\alpha_{2}$ & 1.301 & 0.345 & 1.311 & 0.341 & 1.310 & 0.298 \\
\hline & & & $\beta_{1}$ & 2.298 & 0.621 & 2.313 & 0.602 & 2.205 & 0.495 \\
\hline & & & $\beta_{2}$ & 1.811 & 0.407 & 1.815 & 0.399 & 1.712 & 0.300 \\
\hline & & & $\alpha_{1}$ & 3.415 & 0.956 & 3.415 & 0.949 & 3.364 & 0.711 \\
\hline & & \multirow{3}{*}{$\left(1.5,30,\left(0^{(14)}, 15,0^{(15)}\right)\right)$} & $\alpha_{2}$ & 1.289 & 0.323 & 1.300 & 0.310 & 1.277 & 0.250 \\
\hline & & & $\beta_{1}$ & 2.250 & 0.598 & 2.313 & 0.578 & 2.211 & 0.428 \\
\hline & & & $\beta_{2}$ & 1.783 & 0.385 & 1.811 & 0.370 & 1.719 & 0.287 \\
\hline \multirow{16}{*}{3.0} & \multirow{8}{*}{$(30,35)$} & \multirow{5}{*}{$\left(25,\left(5,0^{(24)}\right)\right)$} & $\alpha_{1}$ & 3.500 & 0.928 & 3.490 & 0.915 & 3.331 & 0.697 \\
\hline & & & $\alpha_{2}$ & 1.233 & 0.301 & 1.288 & 0.299 & 1.215 & 0.210 \\
\hline & & & $\beta_{1}$ & 2.218 & 0.511 & 2.310 & 0.503 & 2.200 & 0.401 \\
\hline & & & $\beta_{2}$ & 1.760 & 0.322 & 1.781 & 0.315 & 1.702 & 0.262 \\
\hline & & & $\alpha_{1}$ & 3.512 & 0.901 & 3.495 & 0.899 & 3.312 & 0.656 \\
\hline & & \multirow{3}{*}{$\left(25,\left(0^{(10)}, 5,0^{(10)}\right)\right)$} & $\alpha_{2}$ & 1.230 & 0.298 & 1.282 & 0.281 & 1.210 & 0.199 \\
\hline & & & $\beta_{1}$ & 2.214 & 0.497 & 2.301 & 0.490 & 2.213 & 0.395 \\
\hline & & & $\beta_{2}$ & 1.752 & 0.303 & 1.777 & 0.307 & 1.713 & 0.249 \\
\hline & \multirow{8}{*}{$(45,45)$} & \multirow{5}{*}{$\left(30,\left(15,0^{(29)}\right)\right)$} & $\alpha_{1}$ & 3.515 & 0.889 & 3.475 & 0.852 & 3.300 & 0.601 \\
\hline & & & $\alpha_{2}$ & 1.217 & 0.265 & 1.225 & 0.256 & 1.212 & 0.187 \\
\hline & & & $\beta_{1}$ & 2.210 & 0.460 & 2.298 & 0.455 & 2.215 & 0.361 \\
\hline & & & $\beta_{2}$ & 1.744 & 0.297 & 1.778 & 0.296 & 1.710 & 0.215 \\
\hline & & & $\alpha_{1}$ & 3.421 & 0.860 & 3.470 & 0.844 & 3.278 & 0.593 \\
\hline & & \multirow{3}{*}{$\left(30,\left(0^{(14)}, 15,0^{(15)}\right)\right)$} & $\alpha_{2}$ & 1.210 & 0.241 & 1.211 & 0.239 & 1.210 & 0.161 \\
\hline & & & $\beta_{1}$ & 2.212 & 0.448 & 2.210 & 0.437 & 2.200 & 0.339 \\
\hline & & & $\beta_{2}$ & 1.737 & 0.281 & 1.755 & 0.277 & 1.712 & 0.203 \\
\hline
\end{tabular}


TABle 4: MILs and PCs of Lomax distributions with $\Phi=(3.0,1.0,2.0,1.5)$.

\begin{tabular}{|c|c|c|c|c|c|c|c|c|c|}
\hline \multirow{2}{*}{$\tau$} & \multirow{2}{*}{$\left(\kappa_{1}, \kappa_{2}\right)$} & \multirow{2}{*}{$(m,(\mathbf{R}))$} & \multirow{2}{*}{$\mathrm{Pa}$} & \multicolumn{2}{|c|}{ ML } & \multicolumn{2}{|c|}{$\mathrm{BMCMC}_{\text {prior }_{0}}$} & \multicolumn{2}{|c|}{$\mathrm{BMCMC}_{\text {prior }_{1}}$} \\
\hline & & & & MILs & PCs & MILs & PCs & MILs & PCs \\
\hline \multirow{16}{*}{1.5} & \multirow{8}{*}{$(35,30)$} & \multirow{5}{*}{$(25,(5,0))$} & $\alpha_{1}$ & 7.235 & $(0.77)$ & 7.027 & $(0.88)$ & 6.548 & $(0.89)$ \\
\hline & & & $\alpha_{2}$ & 3.368 & $(0.88)$ & 3.287 & $(0.88)$ & 3.001 & $(0.90)$ \\
\hline & & & $\beta_{1}^{2}$ & 5.214 & $(0.89)$ & 5.147 & $(0.89)$ & 4.512 & $(0.89)$ \\
\hline & & & $\beta_{2}$ & 4.512 & $(0.89)$ & 4.325 & $(0.90)$ & 3.541 & $(0.90)$ \\
\hline & & & $\alpha_{1}$ & 7.122 & $(0.89)$ & 6.920 & $(0.90)$ & 6.442 & $(0.90)$ \\
\hline & & \multirow{4}{*}{$\left(25,\left(0^{(10)}, 5,0^{(10)}\right)\right)$} & $\alpha_{2}$ & 3.265 & $(0.89)$ & 3.182 & $(0.89)$ & 2.905 & $(0.90)$ \\
\hline & & & $\beta_{1}$ & 5.118 & $(0.91)$ & 5.100 & $(0.91)$ & 4.470 & $(0.89)$ \\
\hline & & & $\beta_{2}$ & 4.410 & $(0.89)$ & 4.360 & $(0.90)$ & 3.480 & $(0.91)$ \\
\hline & \multirow{8}{*}{$(45,45)$} & & $\alpha_{1}$ & 7.001 & $(0.90)$ & 6.780 & $(0.90)$ & 6.311 & $(0.92)$ \\
\hline & & \multirow{4}{*}{$\left(30,\left(15,0^{(29)}\right)\right)$} & $\alpha_{2}$ & 3.170 & $(0.89)$ & 3.165 & $(0.92)$ & 2.798 & $(0.92)$ \\
\hline & & & $\beta_{1}$ & 5.024 & $(0.90)$ & 5.003 & $(0.91)$ & 4.304 & $(0.90)$ \\
\hline & & & $\beta_{2}$ & 4.312 & $(0.90)$ & 4.299 & $(0.91)$ & 3.320 & $(0.91)$ \\
\hline & & & $\alpha_{1}$ & 6.902 & $(0.91)$ & 6.700 & $(0.91)$ & 6.140 & $(0.93)$ \\
\hline & & \multirow{3}{*}{$\left(1.5,30,\left(0^{(14)}, 15,0^{(15)}\right)\right)$} & $\alpha_{2}$ & 3.045 & $(0.90)$ & 3.022 & $(0.92)$ & 2.666 & $(0.92)$ \\
\hline & & & $\beta_{1}$ & 4.920 & $(0.89)$ & 4.901 & $(0.93)$ & 4.123 & $(0.94)$ \\
\hline & & & $\beta_{2}$ & 4.211 & $(0.91)$ & 4.200 & $(0.90)$ & 3.245 & $(0.92)$ \\
\hline \multirow{16}{*}{3.0} & \multirow{8}{*}{$(30,35)$} & \multirow{4}{*}{$\left(25,\left(5,0^{(24)}\right)\right)$} & $\alpha_{1}$ & 6.450 & $(0.90)$ & 6.320 & $(0.90)$ & 5.824 & $(0.90)$ \\
\hline & & & $\alpha_{2}$ & 2.842 & $(0.90)$ & 2.850 & $(0.92)$ & 2.601 & $(0.92)$ \\
\hline & & & $\beta_{1}^{2}$ & 4.754 & $(0.90)$ & 4.701 & $(0.91)$ & 4.013 & $(0.93)$ \\
\hline & & & $\beta_{2}$ & 4.032 & $(0.90)$ & 4.045 & $(0.91)$ & 3.122 & $(0.91)$ \\
\hline & & \multirow{3}{*}{$\left(25,\left(0^{(10)}, 5,0^{(10)}\right)\right)$} & $\alpha_{1}$ & 6.361 & $(0.91)$ & 6.311 & $(0.92)$ & 5.715 & $(0.94)$ \\
\hline & & & $\alpha_{2}$ & 2.715 & $(0.91)$ & 2.711 & $(0.92)$ & 2.514 & $(0.92)$ \\
\hline & & & $\beta_{1}$ & 4.620 & $(0.92)$ & 4.601 & $(0.92)$ & 3.992 & $(0.93)$ \\
\hline & & & $\beta_{2}$ & 3.911 & $(0.90)$ & 3.920 & $(0.93)$ & 3.009 & $(0.93)$ \\
\hline & \multirow{8}{*}{$(45,45)$} & \multirow{4}{*}{$\left(30,\left(15,0^{(29)}\right)\right)$} & $\alpha_{1}$ & 6.155 & $(0.90)$ & 6.140 & $(0.93)$ & 5.560 & $(0.92)$ \\
\hline & & & $\alpha_{2}$ & 2.511 & $(0.94)$ & 2.531 & $(0.92)$ & 2.347 & $(0.92)$ \\
\hline & & & $\beta_{1}$ & 4.413 & $(0.91)$ & 4.422 & $(0.93)$ & 3.870 & $(0.94)$ \\
\hline & & & $\beta_{2}$ & 3.741 & $(0.90)$ & 3.700 & $(0.91)$ & 2.891 & $(0.91)$ \\
\hline & & \multirow{4}{*}{$\left(30,\left(0^{(14)}, 15,0^{(15)}\right)\right)$} & $\alpha_{1}$ & 6.035 & $(0.92)$ & 6.011 & $(0.93)$ & 5.453 & $(0.95)$ \\
\hline & & & $\alpha_{2}$ & 2.415 & $(0.91)$ & 2.427 & $(0.95)$ & 2.215 & $(0.92)$ \\
\hline & & & $\beta_{1}$ & 4.340 & $(0.94)$ & 4.311 & $(0.93)$ & 3.745 & $(0.93)$ \\
\hline & & & $\beta_{2}$ & 3.654 & $(0.92)$ & 3.643 & $(0.92)$ & 2.779 & $(0.96)$ \\
\hline
\end{tabular}

with constructed Monte Carlo simulation studies. The point estimators are measured with mean estimate values (ME) and square rote of simulated variance $(\sqrt{\mathrm{SV}})$ which are given by

$$
\begin{aligned}
& \mathrm{ME}=\bar{\Phi}=\frac{1}{N} \sum_{i=1}^{N} \Phi^{(i)} \\
& \mathrm{SV}=\frac{1}{N} \sum_{i=1}^{N}\left(\Phi^{(i)}-\bar{\Phi}\right)^{2}
\end{aligned}
$$

where $N$ is the iteration number of simulation and $\Phi$ is the parameters' vector. In interval estimation, we adopted probability coverage (PC) and the mean interval length (MIL). In our simulation study, we consider the only set of the values of the parameters $\Phi=\{3.0,1.0,2.0,1.5\}$. A simulation is done with two values of $\tau=\{1.5,3.0\}$ and two choices of prior information and noninformative prior (prior 0), in which posterior distribution is proportional with likelihood function and informative prior (prior 1) the values of prior parameters given by $\{(5.0,1.0),(3.0$, $2.5),(4.0,2.0),(3,2)\}$. The study is carried out under 1000 sets of balanced joint progressive type-I generated of
Lomax lifetime. For each sample, the ML and Bayes estimate is reported, and the Bayesian approach is computed under SEL with 11,000 chains removed the first 1000 as burn-in.

\section{Concluding Remarks}

The commonly used problem in life products with different production lines is measuring the relative merits of the products competing for the duration. The main aim is to use censoring that can be applied to obtain the information in determining time. Recently, a joint censoring scheme and a specially balanced joint censoring scheme can be applied for this problem. BJPHC is proposed for obtaining information about lifetime Lomax products. In this paper, we consider the MLEs of unknown model parameters and the corresponding approximate confidence intervals. Also, we consider Baye's estimation of the unknown parameters under consideration of the gamma priors on the unknown parameters. The Bayes estimates are computed with squared error loss functions. The Bayes estimators' explicit forms cannot be obtained, so the MCMC approach is impalement to get the 
parameter estimation. The results are compared by using Monte Carlo simulation between the performances of the MLEs and approximate Bayes estimates under the assumptions of noninformative and informative priors. The Bayes estimates under the MCMC technique have been required to generate from the posterior distribution. The simulated MCMC sample plot and the corresponding histograms match quite well with the theoretical posterior density functions. The results can be extended to different types of loss functions. The proposed model and the results can also be easily extended for several members of the exponential family. Finally, we should mention that the products in Tables 3 and 4 simulation study show that

(1) The life experiments under balanced jointly progressive type-I censoring scheme present a good source for obtaining the information about Lomax lifetime products

(2) The results improve for larger values of experiment total time $\tau$

(3) The Bayes estimation with informative prior distribution performs better than ML and noninformative prior method

(4) The results of MLE are closed to the Bayes estimates for noninformative prior

(5) For increase, the effective sample size $m$ estimators perform better in the form of $\sqrt{\mathrm{SV}} \mathrm{s}$, interval length, and coverage probability

(6) Censoring scheme with middle censoring performs better than other censoring schemes

(7) The method of MCMC for approximation Bayesian estimate serves well, especially in dimensional cases

\section{Data Availability}

No data were used to support the findings of this study.

\section{Conflicts of Interest}

The authors have no conflicts of interest regarding the publication of the paper.

\section{Acknowledgments}

This study was funded by the Deanship of Scientific Research, Taif University, KSA (research project no. 1-4406179).

\section{References}

[1] A. C. Cohen, "Progressively censored samples in life testing," Technometrics, vol. 5, no. 3, pp. 327-339, 1963.

[2] A. C. Cohen, "Query 18: life testing and early failure," Technometrics, vol. 8, no. 3, pp. 539-549, 1966.

[3] N. Balakrishnan and R. Aggarwala, Progressive Censoring-Theory, Methods, and Applications, Birkh User, Boston, MA, USA, 2000.
[4] N. Balakrishnan and D. Kundu, "Hybrid censoring: models, inferential results and applications," Computational Statistics \& Data Analysis, vol. 57, no. 1, pp. 166-209, 2013.

[5] U. V. R. Rao, I. R. Savage, and M. Sobel, "Contributions to the theory of rank order statistics: the two-sample censored case," The Annals of Mathematical Statistics, vol. 31, no. 2, pp. 415-426, 1960.

[6] K. G. Mehrotra and G. K. Bhattacharyya, "Confidence intervals with jointly type-II censored samples from two exponential distributions," Journal of the American Statistical Association, vol. 77, no. 378, pp. 441-446, 1982.

[7] N. Balakrishnan and A. Rasouli, "Exact likelihood inference for two exponential populations under joint type-II censoring," Computational Statistics \& Data Analysis, vol. 52, no. 5, pp. 2725-2738, 2008.

[8] A. Rasouli and N. Balakrishnan, "Exact likelihood inference for two exponential populations under joint progressive typeII censoring," Communications in Statistics-Theory and Methods, vol. 39, no. 12, pp. 2172-2191, 2010.

[9] A. R. Shafay, N. Balakrishnan, and Y. Abdel-Aty, "Bayesian inference based on a jointly type-II censored sample from two exponential populations," Journal of Statistical Computation and Simulation, vol. 84, no. 11, pp. 2427-2440, 2014.

[10] B. N. Al-Matrafi and G. A. Abd-Elmougod, "Statistical inferences with jointly type-II censored samples from two Rayleigh distributions," Global Journal of Pure and Applied Mathematics, vol. 13, pp. 8361-8372, 2017.

[11] F. A. Momenkhan and G. A. Abd-Elmougod, "Estimations in partially step-stress accelerate life tests with jointly type-II censored samples from Rayleigh distributions," Transylvanian Review, vol. 28, pp. 7609-7616, 2018.

[12] A. Ali, A. M. Almarashi, G. A. Abd-Elmougod, and Z. A. AboEleneen, "Two compound Rayleigh lifetime distributions in analyses the jointly type-II censoring samples," Journal of Mathematical Chemistry, vol. 58, no. 5, pp. 950-966, 2019.

[13] S. Mondal and D. Kundu, "A new two sample type-II progressive censoring scheme," 2016, http://arxiv.org/abs/1609.05805.

[14] S. Mondal and D. Kundu, "Bayesian inference for Weibull distribution under the balanced joint type-II progressive censoring scheme," American Journal of Mathematical and Management Sciences, vol. 39, no. 1, pp. 56-74, 2019.

[15] S. Mondal and D. Kundu, "Inference on Weibull parameters under a balanced two-sample type II progressive censoring scheme," Quality and Reliability Engineering International, vol. 36, no. 1, pp. 1-17, 2020.

[16] K. S. Lomax, "Business failures: another example of the analysis of failure data," Journal of the American Statistical Association, vol. 49, no. 268, pp. 847-852, 1954.

[17] M. Chahkandi and M. Ganjali, "On some lifetime distributions with decreasing failure rate," Computational Statistics \& Data Analysis, vol. 53, no. 12, pp. 4433-4440, 2009.

[18] E. Cramer and A. B. Schmiedt, "Progressively type-II censored competing risks data from lomax distributions," Computational Statistics \& Data Analysis, vol. 55, no. 3, pp. 1285-1303, 2011.

[19] H. A.-Z. Hanaa, "Lifetime competing risks data from Lomax distribution in the presence of accelerates life-testing model with type-I censoring," Journal of Intelligent \& Fuzzy Systems, vol. 38, pp. 2873-2883, 2019.

[20] S. Parsi, M. Ganjali, and N. S. Farsipour, "Conditional maximum likelihood and interval estimation for two Weibull populations under joint type-II progressive censoring," Communications in Statistics-Theory and Methods, vol. 40, no. 12, pp. 2117-2135, 2011. 
[21] D. Kundu and A. Joarder, "Analysis of type-II progressively hybrid censored data," Computational Statistics \& Data Analysis, vol. 50, no. 10, pp. 2509-2528, 2006.

[22] N. Metropolis, A. W. Rosenbluth, M. N. Rosenbluth, A. H. Teller, and E. Teller, "Equation of state calculations by fast computing machines," The Journal of Chemical Physics, vol. 21, no. 6, pp. 1087-1092, 1953.

[23] A. A. Soliman, A. H. Abd Ellah, N. A. Abou-Elheggag, and G. A. Abd-Elmougod, "A simulation-based approach to the study of coefficient of variation of Gompertz distribution under progressive first-failure censoring," Indian Journal of Pure and Applied Mathematics, vol. 42, no. 5, pp. 335-356, 2011.

[24] N. Balakrishnan and R. A. Sandhu, "A simple simulational algorithm for generating progressive type-II censored samples," The American Statistician, vol. 49, no. 2, pp. 229-230, 1995. 